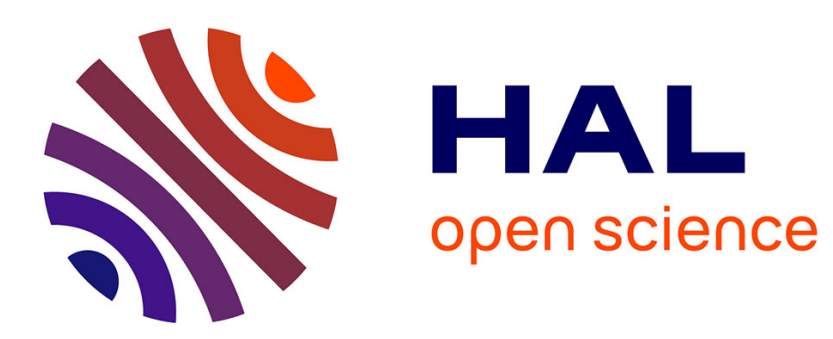

\title{
CALIBRATION DE SONDE INTENSIMÉTRIQUE EN TUBE À ONDE STATIONNAIRE
}

\author{
H. Pépin, T. Phan
}

\section{To cite this version:}

H. Pépin, T. Phan. CALIBRATION DE SONDE INTENSIMÉtRIQUE EN TUBE À ONDE STATIONNAIRE. Journal de Physique IV Proceedings, 1992, 02 (C1), pp.C1-431-C1-434. 10.1051/jp4:1992192 . jpa-00251264

\section{HAL Id: jpa-00251264 https://hal.science/jpa-00251264}

Submitted on 1 Jan 1992

HAL is a multi-disciplinary open access archive for the deposit and dissemination of scientific research documents, whether they are published or not. The documents may come from teaching and research institutions in France or abroad, or from public or private research centers.
L'archive ouverte pluridisciplinaire HAL, est destinée au dépôt et à la diffusion de documents scientifiques de niveau recherche, publiés ou non, émanant des établissements d'enseignement et de recherche français ou étrangers, des laboratoires publics ou privés. 


\title{
CALIBRATION DE SONDE INTENSIMÉTRIQUE EN TUBE À ONDE STATIONNAIRE
}

\author{
H. PEPIN et T.M. PHAN \\ CETIM, Département Acoustique Industrielle, 52 Avenue Félix Louat, BP. 67, F-60300 Senlis cedex, \\ France
}

\begin{abstract}
The performance of acoustic intensimetry using the two-microphone technique depends on the accuracy of the calibration of the measurement system, involving both the probe and the intensity processor.

The recent ISO/IEC 1043 draft standard implies the use of high precision laboratory calibrators for frequency reponse, phase mismatch and directivity measurements.

A calibration method in a standing wave tube has been developped in CETIM with respect to be IEC specifications. Depending on the acoustic field generated in the tube, for a given standing wave ratio, one can measure the intensity probe caracteristics, including diffraction effect and directivity curves.
\end{abstract}

\section{Introduction}

L'intensimétrie acoustique, bien qu'aujourd'hui largement utilisée souffre encore de l'absence de norme définissant de manière rigoureuse les classes instrumentales utilisables. Cette lacune est en passe d'être comblée avec la parution du projet ISO/IEC 1043 (10/91), proposant une classification des appareillages intensimétriques, sondes et processeurs.

Plusieurs méthodes de calibration des sondes intensimétriques, aujourd'hui à gradient de pression "p-p", ont déjà été élaborées ces dernières années : petites cavité, excitation électrośtatique, champ libre. Néanmoins, la nécessité d'un contrôle rigoureux de ces sondes en vue de leur classificatio métrologique requiert des bancs de calibration de laboratoire de grande précision : tolérances en amplitude de l'ordre du dixième de $\mathrm{dB}$, tolérances en phase inférieures au dixième de degré, avec généralisation des résultats en réponse directionnelle (diagramme de directivité).

Le CETIM travaille à la mise au point d'un tel système de calibration consistant en un tube à onde stationnaire à charge active, c'est à dire excité à ses extrémités par deux haut-parleurs contrôlés en amplitude et en phase. Il est ainsi possible de créer dans la zone de mesure au centre du tube, où est suspendue la sonde testée, le type de champ acoustique recherché, caractérisé par son taux d'onde stationnaire.

La détermination des caractéristiques d'une sonde est alors réalisable pour différents types de champ acoustique, propagatif ou réactif, selon que l'on recherche à mettre en évidence, par exemple la diffraction géométrique ou la réponse en phase résiduelle des voies de mesure. 


\section{Principe de fonctionnement du tube et caractéristiques}

Contrairement aux procédures de calibration précédemment mises au point, telles que par excitation électrostatique (hautes et moyennes fréquences) ou en petite cavité (basses fréquences), qui qualifient les voies instrumentales indépendamment de la géométrie de montage des microphones, c'est à dire notamment de l'effet de diffraction de la sonde, l'utilisation du tube à onde stationnaire permet la qualification globale de la sonde testée dans un champ acoustique rigoureusement contrôlé. Outre la diffraction, ce type de mesure permet de tenir compte de l'influence des évents d'égalisation de pression équilibrant la charge arrière des membranes électrostatiques (sondes "p-p" de type "single port").

En tant qu'appareil de précision réservé au laboratoire, le tube est essentiellement utilisé en fréquence pure, les tests étant réitérés pour chaque fréquence de mesure. Son domaine d'utilisation est limité par ses dimensions.

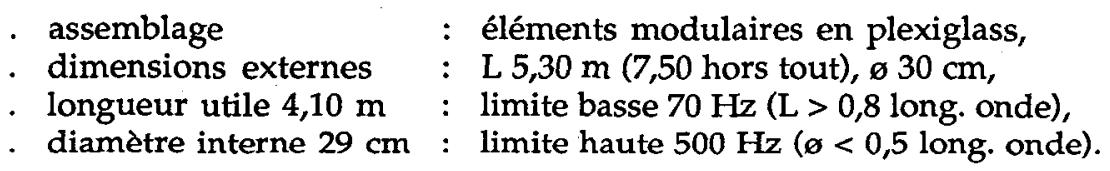

Pour une sonde classique 1/2" de section transversale de l'ordre de $6 \mathrm{~cm}^{2}(\mathrm{Sp})$, la section résultante du tube, $\mathrm{St}=660 \mathrm{~cm}^{2}$, permet le respect de la condition de simulation des ondes planes $\mathrm{St}>=100 \times \mathrm{Sp}$ définie dans le document IEC.

Pour éviter les problèmes de réalisation et d'utilisation d'une terminaison anéchoïque classiquement utilisée sur les tubes de Kundt, le tube CETIM comporte deux haut-parleurs montés en vis à vis à chacune de ses extrémités et contrôlés en amplitude et en phase. On peut ainsi recréer les conditions de fonctionnement recherchées (ondes planes, taux d'onde stationnaire), via le paramètrage des signaux d'excitation appliqués à chacun d'eux, notamment en imposant un déphasage fixe prédéfini.

Enfin, de manière à éviter les problèmes mécaniques et métrologiques liés au montage d'une sonde mobile à l'intérieur du tube, la sonde testée est suspendue rigidement selon son axe vertical de rotation au centre du dispositif. La description spatiale de l'onde acoustique peut alors être réalisée par déplacement des hautparleurs, mobiles à chaque extrémité du tube, ce qui permet de simuler le déplacement correspondant de la sonde dans le champ acoustique créé. La plage d'excursion relative sonde - haut-parleurs, d'environ $1 \mathrm{~m}$, est réglable avec une précision de l'ordre du dixième de $\mathrm{mm}$ à l'aide d'un système de positionnement robotisé à moteurs pas à pas. Le positionnement angulaire de la sonde autour de son axe vertical reste quant à lui manuel, avec une précision de l'ordre du demi degré.

Les figures 1 et 2 donnent un schéma général du dispositif ainsi qu'une illustration du montage de la sonde au centre du tube. 

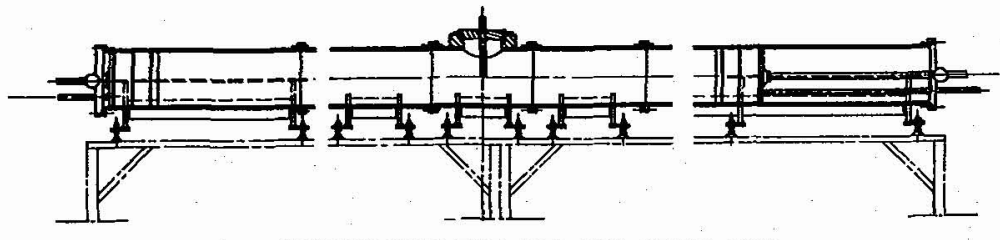

STANDIMG WAVE TUBE WITH TWO MOVING ENDS



\section{Figure 1}

Figure 2

III. Système de contrôle et de mesure

Le système de commande utilisé est réalisé autour d'un analyseur FFT multivoies (HP $3566 \mathrm{~A}$ ) disposant de son propre générateur de bruit. Il permet selon le type de montage le suivi de cinq voies de mesure (extensibles à huit) : signal source, signaux d'excitation amplifiés (haut-parleurs), signaux délivrés par la sonde. La plage de mesure normalisée correspond au domaine $50 \mathrm{~Hz}-6.3 \mathrm{kHz}$, en fréquence pure ou en bruit large bande filtré (bruit rose par bande tiers d'octave). L'amplification et le contrôle en phase des haut-parleurs est assuré par des appareillages indépendants connectés en sortie du générateur.

\section{Mesures du champ acoustique dans le tube}

Les figures 3 et 4 donnent des exemples de description du champ acoustique Pression/Intensité au centre du tube, obtenus à la fréquence $300 \mathrm{~Hz}$, pour différentes valeurs du taux d'onde stationnaire (déplacement des HP sur environ $60 \mathrm{~cm}$ ). La figure 5 montre l'influence de la correction en phase de la sonde dans la mesure de l'intensité pour un faible taux d'onde stationnaire (sonde électrostatique AKSUD 1/2", TOS \# 0.1)

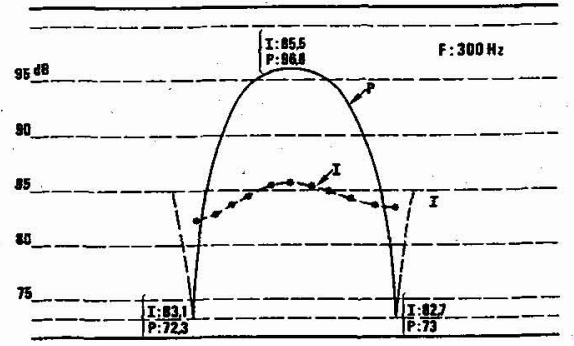

Figure 3

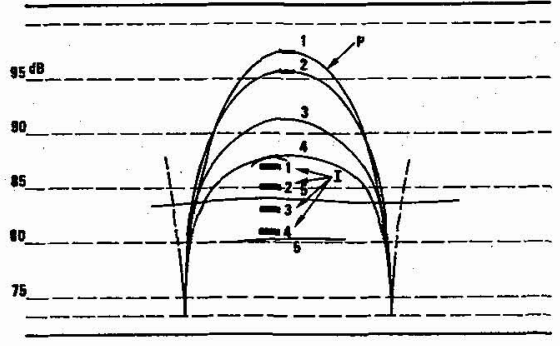

Figure 4 


\section{Exemples de mesure en fonction de l'onde d'incidence}

La nature "vectorielle" de la mesure intensimétrique rend indispensable la connaissance des caractéristiques directionnelles des sondes, notamment lorsque l'on s'écarte de l'incidence nulle vers la zone de sensibilité minimale, où ne subsiste que l'intensité résiduelle de phase. La figure 6 illustre les résultats obtenus avec une sonde appariée classique (BK 3519) en faisant varier l'incidence par bonds de 30 degrés : niveaux de pression, intensité mesurée, intensité théorique (loi en cosinus). On y constate une bonne convergence théorie-mesure, avec des écarts toujours inférieurs au $1 / 2 \mathrm{~dB}$, souvent inférieurs ou égaux au dixième de $\mathrm{dB}$.

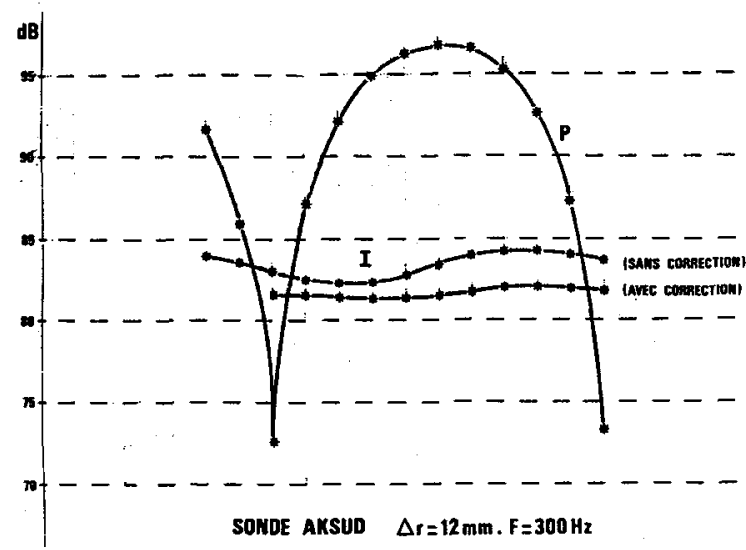

Figure 5

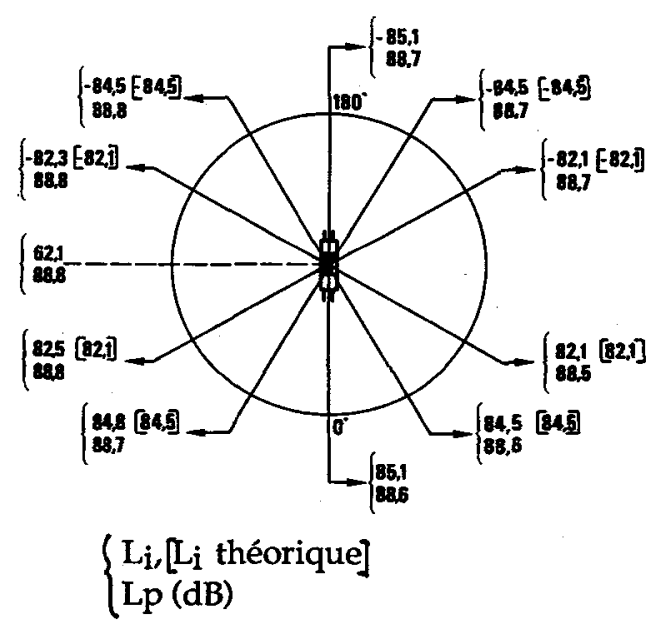

Figure 6

\section{Conclusion}

Malgré un encombrement important et une mise en oeuvre délicate, le tube à onde stationnaire permet la réalisation de calibration de grande précision, nécessaire pour les tests de réception et de classification des sondes intensimétriques. Confiné à un usage de laboratoire, il constitue néanmoins un élément indispensable à l'élargissement de la diffusion de l'intensimétrie vers l'ensemble des mesures normalisées.

\section{Remerciements}

L'étude ayant conduit à l'élaboration du tube de calibration fait partie d'un programme de recherche européen supervisé par le Bureau Communautaire de Référence. Le prolongement de cette étude concernant la mise au point de procédures complètes de calibration de sonde en champ libre ou en tube à onde stationnaire est réalisé dans le cadre d'une convention de recherche du Bureau National de Métrologie. 